\title{
Home truths around Jupiter
}

\section{C.T. Russell}

Physics of the Jovian Magnetosphere.

Edited by A.J. Dessler.

Cambridge University Press: 1983.

Pp.540. £25, \$29.50.

EARLY in the era of space exploration we sent probes to the planets simply to discover what was there. Later, as the glamour of space exploration faded somewhat, it became fashionable (or perhaps necessary) to rationalize our quest to understand the planets in terms of improving our understanding of the Earth.

But how can you learn anything about the terrestrial magnetosphere from Jupiter? The Jovian magnetosphere has volcanoes in it, spewing out sulphur which becomes ionized and spun up by the rapid rotation of the planet. This rapidly rotating plasma stretches the Jovian field so that the magnetosphere is more disc-like than spherical. By contrast centrifugal force in the terrestrial plasma has an insignificant effect on the position of the magnetopause. Jupiter emits powerful radio waves, too, and releases energetic electrons which in some energy ranges dominate solar electrons at the Earth many AU away. In addition to this, it has radiation belts which roast our spacecraft if they linger too close to the planet.

We do learn of course. We learn basic plasma physics; we gain insight into how astrophysical plasmas may behave; we gain an appreciation of processes which operate on less grand scales in the terrestrial magnetosphere. And there are some processes that operate almost identically on the two planets, such as ELF and VLF emissions. On Earth, we cannot easily determine how much man's activity on the surface of the planet affects the generation of these emissions. The observations of Jupiter made so far indicate that these waves do, in fact, originate spontaneously in the plasma.

There is, however, still much to be learned, both from Jupiter and about it. Indeed the very structure of The Physics of the Jovian Magnetosphere reflects the immaturity of the discipline. There are eight observational papers centred around specific measurement techniques (magnetic fields, low energy particles, radio emissions, etc.) and four papers in which theorists discuss their favourite ideas. In a more mature phase of our understanding, such a book would have a series of articles discussing physical processes each of which covered all the relevant observations and theories. But we won't reach that stage for quite some time.

In the meantime this book will have to suffice. The authors have written sound, complete articles, not just the extended versions of oral presentations that you often find in conference proceedings. These papers have been carefully reviewed and the contributors, perhaps with the editor's encouragement, have responded well. As a result the book is safe for firstyear graduate students and others uninitiated to the sometimes intense rivalries that have developed as resources for such research have declined. The book would, however, have benefited from a discussion of satellite atmospheres and ionospheres (such a review is to be found in The Satellites of Jupiter, edited by D. Morrison and

\section{Venusian probes}

\section{Lionel Wilson}

\section{Venus.}

Edited by D.M. Hunten, L. Colin, T.M. Donahue and V.I. Moroz.

University of Arizona Press: 1983.

$$
\text { Pp.1,143. \$49.95. }
$$

CONSIDERING its similarity in size to the Earth and its obvious importance in understanding the origin and evolution of the silicate planets, it is particularly unfortunate that Venus is so well equipped (by its opaque, dense, hot atmosphere) to hide its surface from our probes. However, technological persistence pays off. To date, a total of thirteen Soviet and five American missions have visited Venus; the combined efforts have resulted in fifteen surface landers (some soft, some hard), seven flybys and three orbiters which has led to a steady increase in our knowledge of the planet.

A conference held in California in November 1981 provided the opportunity for a detailed review of Venus studies (see Nature 296, 13-20; 1982), and this book consists of much of the material presented at the meeting together with numerous papers submitted later by Soviet authors. As a result, the collection gives a wellbalanced impression of the total effort being directed at Venus. It is interesting that a number of the papers are co-authored by Eastern-bloc and Western scientists, symbolic of an encouragingly high level of scientific collaboration. So great has been the upsurge of interest in Venus, particularly since the arrival in orbit of the US Pioneer Venus Orbiter in 1978, that the conference volume could not find room for all of the papers submitted - the interested published earlier this year by the University of Arizona Press).

The book ends with three appendices, a single list of references and an index. The first is a list of symbols and acronyms which was very handy in reading the book. The second, especially welcome, discusses coordinate systems; Dessler's jovial disposition is apparent in this attempt to prevent Jovian mispositions. The third appendix lists selected physical parameters of Jupiter and Io. Finally, there is the index, essential for any book of this type but all too often forgotten.

In short this is noc a run-of-the-mill conference proceedings' volume, but a valuable thorough account of what we know - and don't know - about Jupiter's magnetosphere.

C.T. Russell is Professor of Geophysics and Space Physics at the University of California, Los Angeles.

reader should also consult the special Venus issues of the journal Icarus dated August and November 1982.

A few of the papers in this volume are devoted to useful historical summaries; the rest are divided 14:6:5 among the atmosphere, ionosphere and surface, respectively. This emphasizes the leading role that studies of the atmosphere have played so far in the investigation of the evolution of Venus, and underlines the great amount of work remaining to be done on the chemical and physical structure of the lithosphere, the thermal state of the interior, and the nature of the tectonic regimes that represent the interaction between these two systems. Such studies are progressing steadily as a synthesis takes place between the surface chemical analyses made by the softlanders Veneras 13 and 14 and the radar topography, roughness and dielectric constant data obtained by the Pioneer Venus Orbiter and by Earth-based radar measurements. However, one feels that it will be some time before our understanding of the surface and interior matches that of the atmosphere.

With the inclusion of a paper summarizing the Venera 13 and 14 measurements, made after the conference, this volume has the distinction of being a particularly upto-date representation of our knowledge of Venus. However, two more Soviet probes are even now en route to the planet, and the US Venus Radar Mapper probe is expected to provide a very great increase in our knowledge of the surface when it begins measurements in mid-1988. So with respect to the surface, we can assume that the best is yet to come.

Lionel Wilson is Head of the Planetary Sciences Section of the Department of Environmental Sciences at Lancaster University. 